\title{
Sequential sampling in clinical cytogenetics: a quality control viewpoint
}

\author{
MIGUEL A DE ARCE AND SUSAN P MCMANUS \\ From the Children's Hospital, Temple Street, Dublin 1, Ireland.
}

SUMmARY We have observed that there is some resemblance between the problems of sampling in industrial quality control and the process of diagnosis of mixed cell populations in cytogenetics. This resemblance enabled us to draw from the methodology of the former science to solve some diagnostic problems in the latter. We considered which of the several sampling procedures available for quality control would be more efficient and more suitable in clinical cytogenetics, concluding that 'sequential sampling' combines both features. We also studied the effect that some abnormalities due to technical factors had on sample size required to reach a diagnosis with a given level of confidence. We give a set of tables for sequential sampling in diagnostic laboratories, illustrating their use in discriminating between mosaicism and pseudomosaicism in prenatal diagnosis and in the diagnosis of the fragile $\mathrm{X}$ syndrome. Finally, we discuss the merits of sequential sampling as shown here, comparing it with the current method used in cytogenetics to exclude chromosomal mosaicism.

Verification of the existence of a second cell line in a clinical sample is a problem commonly found in the cytogenetic diagnosis of several pathological situations. It occurs in blood cultures and in prenatal diagnosis, if mosaicism is being considered. It has been suggested ${ }^{1}$ that the cytogenetic diagnosis of fragile $\mathrm{X}$ presents a similar problem, since patients typically express the abnormal chromosome only in a proportion of their cells. In the diagnosis of leukaemia from bone marrow aspirates, the purpose is again to determine the presence of possible pathological clones identifiable by numerically or structurally abnormal karyotypes. These are 'pathological factors' that occur with a mixed cell population and obviously they pose a sampling problem, because if the size of the sample analysed is too small, the second line is likely to be missed.

The problem stated above is compounded by the fact that sometimes the growth medium, or poorly understood biological or technical factors, can provoke low frequencies of chromosomal abnormalities that mimic pathological cases. For instance, it would appear that culture conditions suitable for the expression of fragile $\mathrm{X}$ induce telomeric chromosomal changes on the $\mathrm{X}$ chromosome of normal subjects. ${ }^{2-4}$ There are random chromosome losses, the frequency and type of which can be predicted as

Received for publication 9 June 1987.

Revised version accepted for publication 25 September 1987. a function of size and other chromosomal parameters, in lymphocyte cultures from young adults. ${ }^{5}$ An age related factor is involved in the spontaneous loss of one $\mathrm{X}$ chromosome from cultured lymphocytes of normal women. ${ }^{6}$ 'Single abnormal cells' are randomly distributed in amniotic fluids, so that their frequency can be used to predict accurately the expected numbers of cases of pseudomosaicism and mosaicism when a particular method of harvesting is in operation. ${ }^{7}$ Lastly, abnormalities could occur through accidents in the process of making the slides. Here we will refer to all of these as 'technical factors', as opposed to the 'pathological factors' mentioned above. We see then that in cases where 'technical factors' are known to occur, taking a sample that is too large would increase the probability of including an abnormal cell produced by these, yielding a false positive result.

From the point of view of sampling, cytogeneticists are faced in these situations with a problem similar to the one confronted in industrial quality control. The object of this science is to sentence a production batch as acceptable or unacceptable by a potential customer, having established an upper limit (or acceptance number) to the number of defective items that are attributable to random variations of the production process and a lower limit (or rejection number) to the number of defectives that would indicate a fault in the production process. In cytogenetics, the problem is to diagnose a culture as 
normal or pathological having established an upper limit to the frequency of abnormal metaphases that can be expected due to 'technical factors', and a lower limit to the frequency of abnormal metaphases that indicate 'genuine pathology'.

In both situations a sampling plan is required. Deciding on a sampling plan entails (1) selection of a sampling procedure, (2) determination of sample size, and (3) determination of acceptance and rejection numbers. Only one aspect of the second of these steps, that is, the sample size that allows exclusion of chromosomal mosaicism with a given level of confidence, has been dealt with in clinical cytogenetics, ${ }^{89}$ with some modifications for the case of amniotic fluids harvested by the flask method. ${ }^{10}$ In those references, consideration is not given to the effect of low frequencies of abnormal karyotypes due to the 'technical factors' mentioned above. The purpose of this paper is to draw from the methodology of industrial quality control to suggest the sampling procedure that would be most efficient and suitable in diagnostic cytogenetics, considering successively the three steps: procedure, sample size, and rules for acceptance and rejection.

\section{Methods}

We include the minimum amount of terminology, references, and formulae required to derive sampling plans or repeat calculations for conditions applying in any laboratory.

\section{CONSTRAINTS}

$\mathrm{p}_{1}$ is the proportion of a second cell line occurring as an artefact (technical factors) even in normal samples. $(\alpha)$ is the probability of diagnosing as pathological a sample expressing $\mathrm{p}_{1} \%$ abnormal cells only. The value of this probability may be at $5 \%$. (1- $\alpha)$ is then the probability of accepting such a sample as normal.

$\mathrm{p}_{2}$ is the proportion of second cell lines of abnormal karyotype above which we will consider the sample as a pathological mixture. $(\beta)$ is the probability of taking as normal a sample expressing $\mathrm{p}_{2} \%$ abnormal cells. The value of this probability may be set at $5 \%$ or $10 \%$ and $(1-\beta)$ is then the probability of diagnosing such a sample as pathological.

From the definitions above it can be gathered that to decide on a sampling plan from a particular situation and technique we require to know $p_{1}$ and $\mathrm{p}_{2}$ and decide on $\alpha$ and $\beta$.

\section{SAMPLING PROCEDURES}

\section{Single sampling}

If we were to establish as a guideline for a laboratory that from all blood samples of males queried as $\stackrel{\mathbb{D}}{?}$ fragile X 100 metaphases should be scored, we $\vec{F}$ would have settled for single sampling with $N_{1}=100$.

\section{Double and multiple sampling}

In the previous example, we could establish instead $\frac{\overline{\bar{p}}}{\bar{\phi}}$ that two smaller samples of size $\mathrm{N}_{2}<\mathrm{N}_{1}$ should be $\stackrel{\mathbb{\Omega}}{\varrho}$ studied from each queried fragile $X$ male. In this case we are doing double sampling. We could decide $\overrightarrow{0}$ this for three reasons: firstly to prevent the tediousness of scanning a large sample, secondly because $\vec{\omega}$ we may hope, at least in some cases, that time could? be saved because a diagnosis could be reached with the first sample alone, and lastly, because of ${ }_{i}$ uncertainties attaching to the culture methods, we might wish to examine more than one culture combination. We could also decide on three sam- 8 ples of size $\mathrm{N}_{3}<\mathrm{N}_{2}$, four samples of size $\mathrm{N}_{4}<\mathrm{N}_{3}$, and $\mathrm{O}$ so on. This would be multiple sampling.

\section{Sequential sampling}

Still with the example of the fragile $X$, we could $\stackrel{\mathbb{D}}{D}$ decide not to have a fixed sample size but to analyse $\frac{\sigma}{0}$ metaphase by metaphase, and decide on a diagnosis $\mathbb{Q}$ of the whole culture as soon as evidence is suffi- $\overrightarrow{0}$ ciently strong one way or the other. This is known $\$$ sequential sampling and its mathematical develo ment is due to Wald. ${ }^{11}$

SAMPLE SIZE AND ACCEPTANCE AND REJECTION NUMBERS

Regrettably, there are no simple formulae to deter- $\stackrel{\mathbb{Q}}{2}$ mine sample size or acceptance and rejection $\overrightarrow{\overrightarrow{0}}$ numbers for single or double sampling. However, 3 having decided on the constraints involved in a particular problem, and on the sampling procedure, sample sizes and acceptance and rejection numbers can be determined by consulting the corresponding $\stackrel{0}{0}$ tables from references 12 and 13 for single and double sampling respectively, or reference 14 for all

TABLE 1 Master table for the selection of acceptable errors.

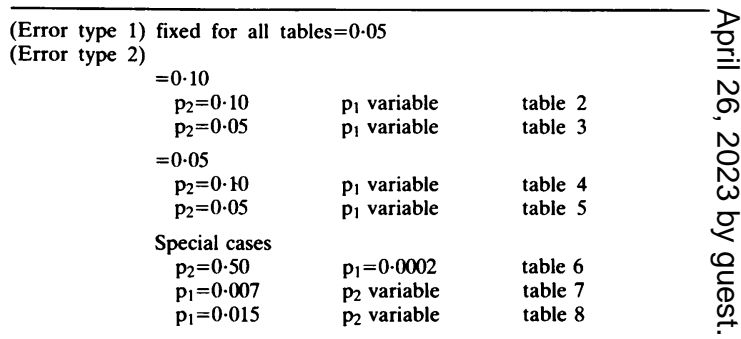

Error type $1=$ probability of diagnosing as pathological a sample expressing 7 $\mathrm{p}_{1} \%$ abnormal cells (same abnormality), which are due to technical factors. Error type $2=$ probability of diagnosing as normal a sample expressing $p \%$ (D) abnormal cells (same abnormality), which are due to pathological factors. 
Sequential sampling in clinical cytogenetics: a quality control viewpoint

TABLE 2 Acceptance and rejection numbers in sequential sampling from mixed cell populations.

\begin{tabular}{|c|c|c|c|c|c|c|c|c|c|c|c|}
\hline \multicolumn{12}{|c|}{$\alpha=0.05, \quad \beta=0.10, p_{2}=0.10$} \\
\hline \multicolumn{3}{|l|}{$\begin{array}{l}p_{I}=0.05 \\
A S S=120\end{array}$} & \multicolumn{3}{|c|}{$\begin{array}{l}p_{I}=0.04 \\
A S S=71\end{array}$} & \multicolumn{3}{|c|}{$\begin{array}{l}p_{I}=0.03 \\
A S S=55\end{array}$} & \multicolumn{3}{|c|}{$\begin{array}{l}p_{1}=0.02 \\
A S S=39\end{array}$} \\
\hline$A$ & & & $B$ & & & $C$ & & & $D$ & & \\
\hline$m$ & $c$ & $d$ & $m$ & $c$ & $d$ & $m$ & $c$ & $d$ & $m$ & $c$ & $d$ \\
\hline 16 & - & 6 & 16 & - & 5 & 12 & - & 4 & 6 & - & 3 \\
\hline 29 & - & 7 & 31 & - & 6 & 30 & 0 & 5 & 25 & 0 & 4 \\
\hline 42 & 0 & 7 & 35 & 0 & 6 & 47 & 1 & 6 & 44 & 1 & 5 \\
\hline 43 & 0 & 8 & 46 & 0 & 7 & 64 & 2 & 7 & 63 & 2 & 6 \\
\hline 56 & 1 & 8 & 50 & 1 & 7 & 81 & 3 & 8 & 82 & 3 & 7 \\
\hline 58 & 1 & 9 & 61 & 1 & 8 & 98 & 4 & 9 & 101 & 4 & 8 \\
\hline 69 & 2 & 9 & 65 & 2 & 8 & 116 & 5 & 10 & 120 & 5 & 9 \\
\hline 71 & 2 & 10 & 76 & 2 & 9 & 132 & 6 & 11 & 139 & 6 & 10 \\
\hline 83 & 3 & 10 & 80 & 3 & 9 & 102 & 0 & 11 & 107 & 0 & 10 \\
\hline 85 & 3 & 11 & 92 & 3 & 10 & & & & & & \\
\hline 97 & 4 & 12 & 96 & 4 & 10 & & & & & & \\
\hline 111 & 5 & 12 & 107 & 4 & 11 & & & & & & \\
\hline 113 & 5 & 13 & & & & & & & & & \\
\hline 125 & 6 & 13 & & & & & & & & & \\
\hline 127 & 6 & 14 & & & & & & & & & \\
\hline 138 & 7 & 14 & & & & & & & & & \\
\hline $\begin{array}{l}p_{1}=0.01 \\
A S S=28\end{array}$ & & & $p_{l}=0$ & & & $p_{I}=0$ & & & $p_{l}=0$ & & \\
\hline$A S S=28$ & & & ASS & & & $A S S$ & & & $A S S$ & & \\
\hline$E$ & & & $F$ & & & $G$ & & & $H$ & & \\
\hline$m$ & $c$ & $d$ & $m$ & $c$ & $d$ & $m$ & $c$ & $d$ & $m$ & $c$ & $d$ \\
\hline 20 & - & 3 & 22 & - & 3 & 23 & 0 & - & 23 & 0 & - \\
\hline 23 & 0 & 3 & 23 & 0 & 3 & 24 & 0 & 3 & 27 & 0 & 3 \\
\hline 45 & 0 & 4 & 48 & 0 & 4 & 50 & 1 & 3 & 51 & 1 & 3 \\
\hline 49 & 1 & 4 & 49 & 1 & 4 & 51 & 1 & 4 & 55 & 1 & 4 \\
\hline 70 & 1 & 5 & 74 & 1 & 5 & 77 & 2 & 4 & 79 & 2 & 4 \\
\hline 74 & 2 & 5 & 75 & 2 & 5 & 78 & 2 & 5 & 83 & 2 & 5 \\
\hline 95 & 2 & 6 & 100 & 2 & 6 & 104 & 3 & 5 & 107 & 3 & 5 \\
\hline 99 & 3 & 6 & 101 & 3 & 6 & 105 & 3 & 6 & & & \\
\hline 120 & 3 & 7 & 125 & 3 & 7 & & & & & & \\
\hline 124 & 4 & 7 & & & & & & & & & \\
\hline 145 & 4 & 8 & & & & & & & & & \\
\hline $\begin{array}{l}p_{I}=0.006 \\
A S S=24\end{array}$ & & & $\begin{array}{l}p_{1}= \\
A S\end{array}$ & & & $\begin{array}{l}p_{1}= \\
A S S\end{array}$ & & & $\begin{array}{l}p_{I}= \\
A S S\end{array}$ & & \\
\hline$I$ & & & $J$ & & & $K$ & & & $L$ & & \\
\hline$m$ & $c$ & $d$ & $m$ & $c$ & $d$ & $m$ & $c$ & $d$ & $m$ & $c$ & $d$ \\
\hline 23 & 0 & 2 & 22 & - & 2 & 4 & - & 2 & 7 & - & 2 \\
\hline 30 & 0 & 3 & 33 & 0 & 3 & 22 & 0 & 2 & 22 & 0 & 2 \\
\hline 52 & 1 & 3 & 53 & 1 & 3 & 37 & 0 & 3 & 42 & 0 & 3 \\
\hline 59 & 1 & 4 & 64 & 1 & 4 & 55 & 1 & 3 & 57 & 1 & 3 \\
\hline 81 & 2 & 4 & 84 & 2 & 4 & 70 & 1 & 4 & 78 & 1 & 4 \\
\hline 88 & 2 & 5 & 95 & 2 & 5 & 88 & 2 & 4 & 93 & 2 & 4 \\
\hline 111 & 3 & 5 & 115 & 3 & 5 & 103 & 2 & 5 & 113 & 2 & 5 \\
\hline 118 & 3 & 6 & 126 & 3 & 6 & 121 & 3 & 5 & 128 & 3 & 5 \\
\hline 134 & 4 & 6 & 146 & 4 & 6 & 135 & 3 & 6 & 148 & 3 & 6 \\
\hline 147 & 4 & 71 & 156 & 4 & 7 & 153 & 4 & 6 & & & \\
\hline 169 & 5 & 72 & 177 & 5 & 7 & & & & & & \\
\hline 176 & 5 & 82 & & & & & & & & & \\
\hline $\begin{array}{l}p_{1}=0.002 \\
A S S=21\end{array}$ & & & $\begin{array}{l}p_{1}= \\
A S\end{array}$ & & & $\begin{array}{l}p_{1}= \\
A S S\end{array}$ & & & $\begin{array}{l}p_{1}= \\
A S S\end{array}$ & & \\
\hline$M$ & & & $N$ & & & $O$ & & & $P$ & & \\
\hline$m$ & $c$ & $d$ & $m$ & $c$ & $d$ & $m$ & $c$ & $d$ & $m$ & $c$ & $d$ \\
\hline 11 & - & 2 & 17 & - & 2 & 21 & 0 & - & 21 & 0 & - \\
\hline 22 & 0 & 2 & 22 & 0 & 2 & 23 & 0 & 2 & 33 & 0 & 2 \\
\hline 50 & 0 & 3 & 62 & 0 & 3 & 73 & 1 & 2 & 82 & 1 & 2 \\
\hline 61 & 1 & 3 & 67 & 1 & 3 & 76 & 1 & 3 & 93 & 1 & 3 \\
\hline 88 & 1 & 4 & 108 & 1 & 4 & 125 & 2 & 3 & 141 & 2 & 3 \\
\hline 100 & 2 & 4 & 112 & 2 & 4 & 127 & 2 & 4 & 153 & 2 & 4 \\
\hline 128 & 2 & 5 & 153 & 2 & 5 & 176 & 3 & 4 & & & \\
\hline 139 & 3 & 5 & 157 & 3 & 5 & & & & & & \\
\hline 167 & 3 & 6 & & & & & & & & & \\
\hline 177 & 4 & 6 & & & & & & & & & \\
\hline
\end{tabular}

(-) means that no decision can be made as to normal (c) or pathological (d). For interpretation of other symbols, see text. 
procedures. By definition, in sequential sampling there is no fixed sample size (m), and instead a diagnosis is reached only on the basis of acceptance and rejection numbers, which in this case are determined as follows.

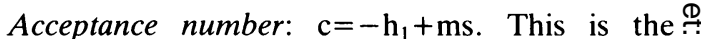
maximum number of abnormal cells which can be $\overrightarrow{\vec{F}}$ accepted as being associated with 'technical factors'.

Rejection number: $\mathrm{d}=\mathrm{h}_{2}+\mathrm{ms}$. This is the minimum number of abnormal cells which would lead to

TABLE 3 Acceptance and rejection numbers in sequential sampling from mixed cell populations.

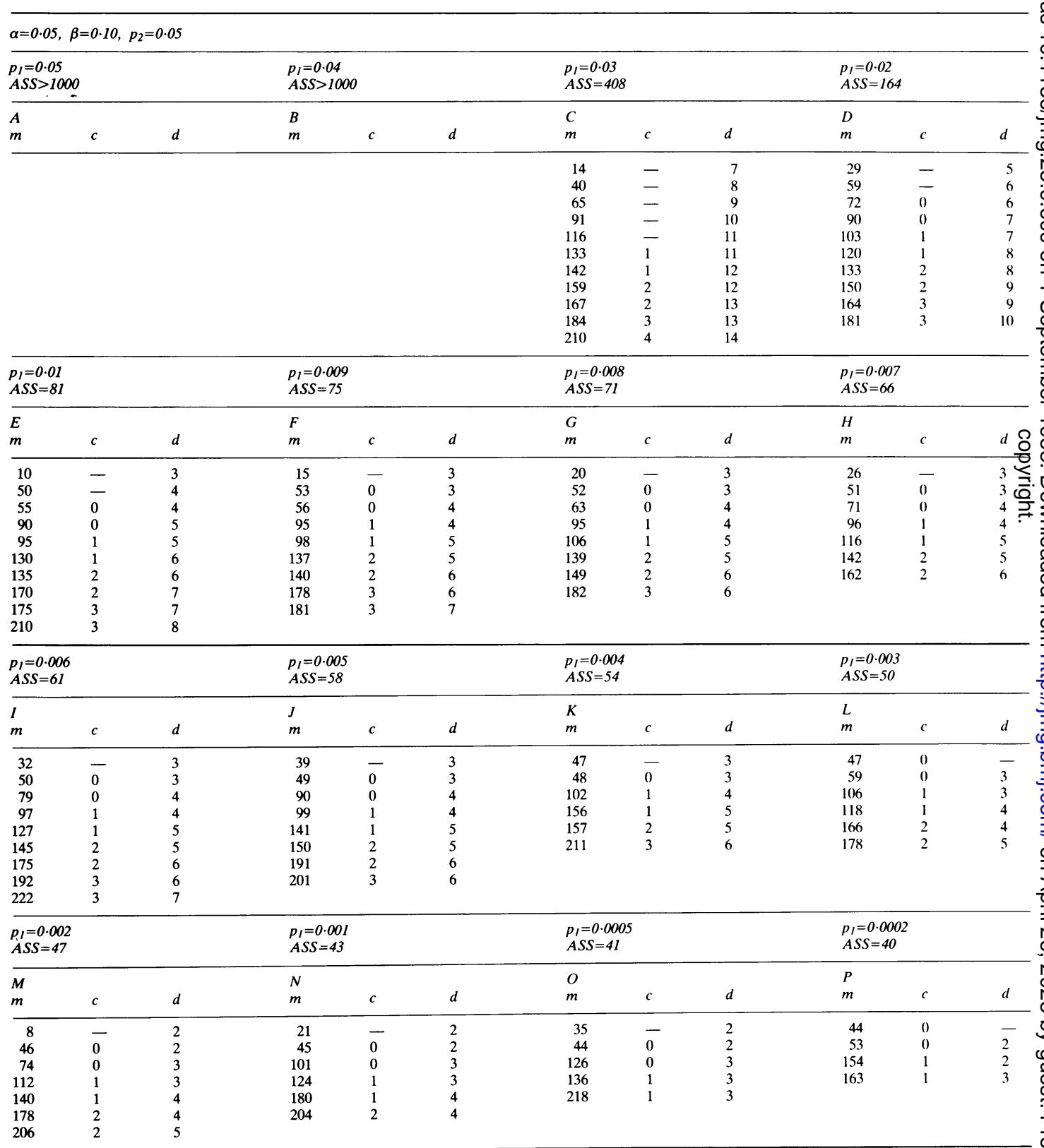

(-) means that no decision can be made as to normal (c) or pathological (d). The tables left blank give average samples sizes that are too large. For interpretation of other symbols, see text. 
rejection of the hypothesis that only 'technical factors' are involved. In the above equations,

$$
\begin{aligned}
& \mathrm{h}_{1}=\mathrm{b} /\left(\mathrm{g}_{1}+\mathrm{g}_{2}\right) \\
& \mathrm{h}_{2}=\mathrm{a} /\left(\mathrm{g}_{1}+\mathrm{g}_{2}\right)
\end{aligned}
$$

$$
\begin{aligned}
& \mathrm{s}=\mathrm{g}_{2} /\left(\mathrm{g}_{1}+\mathrm{g}_{2}\right) \\
& \mathrm{a}=1 \mathrm{n}[(1-\beta) / \alpha] \\
& \mathrm{b}=\ln [(1-\alpha) / \beta] \\
& \mathrm{g}_{1}=\ln \left(\mathrm{p}_{2} / \mathrm{p}_{1}\right)
\end{aligned}
$$

TABLE 4 Acceptance and rejection numbers in sequential sampling from mixed cell populations.

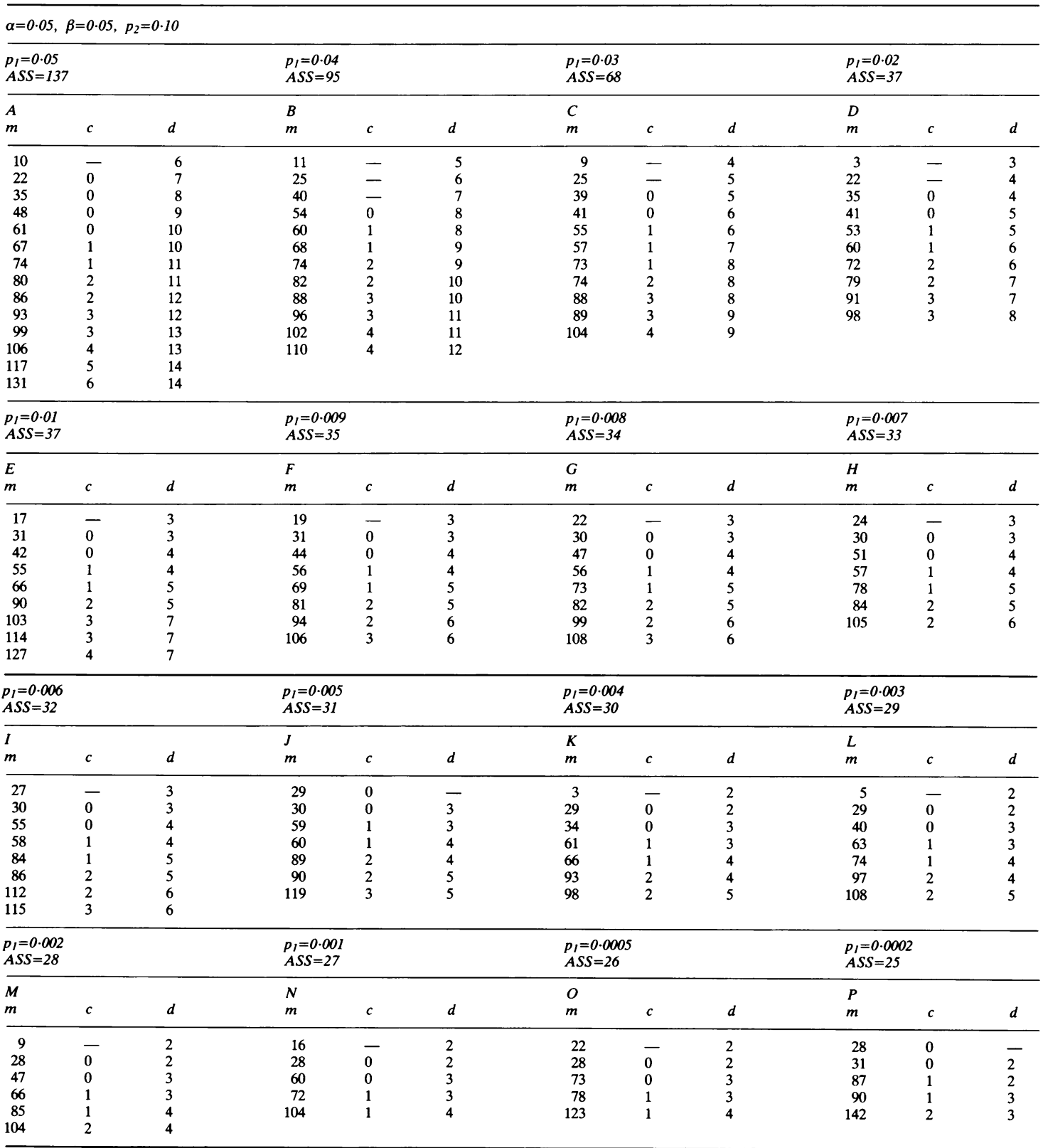

(-) means that no decision can be made as to normal (c) or pathological (d). For interpretation of other symbols, see text. 


$$
\mathrm{g}_{2}=1 \mathrm{n}\left[\left(1-\mathrm{p}_{1}\right) /\left(1-\mathrm{p}_{2}\right)\right]
$$

and $\mathrm{p}_{1}, \mathrm{p}_{2}, \alpha$, and $\beta$ are as defined above.

(1) for $\mathrm{p}=0, \mathrm{~m}_{\mathrm{p} 0}=\mathrm{h}_{1} / \mathrm{s}$

To sketch the function expressing the average sample size in sequential sampling, Wald ${ }^{11}$ gives the following five pairs of values:

(2) for $\mathrm{p}=\mathrm{p}_{1}, \mathrm{~m}_{\mathrm{p} 1}=\left[(1-\alpha) \mathrm{h}_{1}-\mathrm{h}_{2} \alpha\right] /\left(\mathrm{s}-\mathrm{p}_{1}\right)$

(3) for $\mathrm{p}=\mathrm{s}, \mathrm{m}_{\mathrm{s}}=\mathrm{h}_{1} \cdot \mathrm{h}_{2} / \mathrm{s}(1-\mathrm{s})$

(4) for $p=p_{2}, m_{p 2}=\left[(1-\beta) h_{2}-h_{1} \cdot \beta\right] /\left(p_{2}-s\right)$

(5) for $\mathrm{p}=1, \mathrm{~m}_{1}=\mathrm{h}_{2} /(1-\mathrm{s})$.

TABLE 5 Acceptance and rejection numbers in sequential sampling from mixed cell populations.

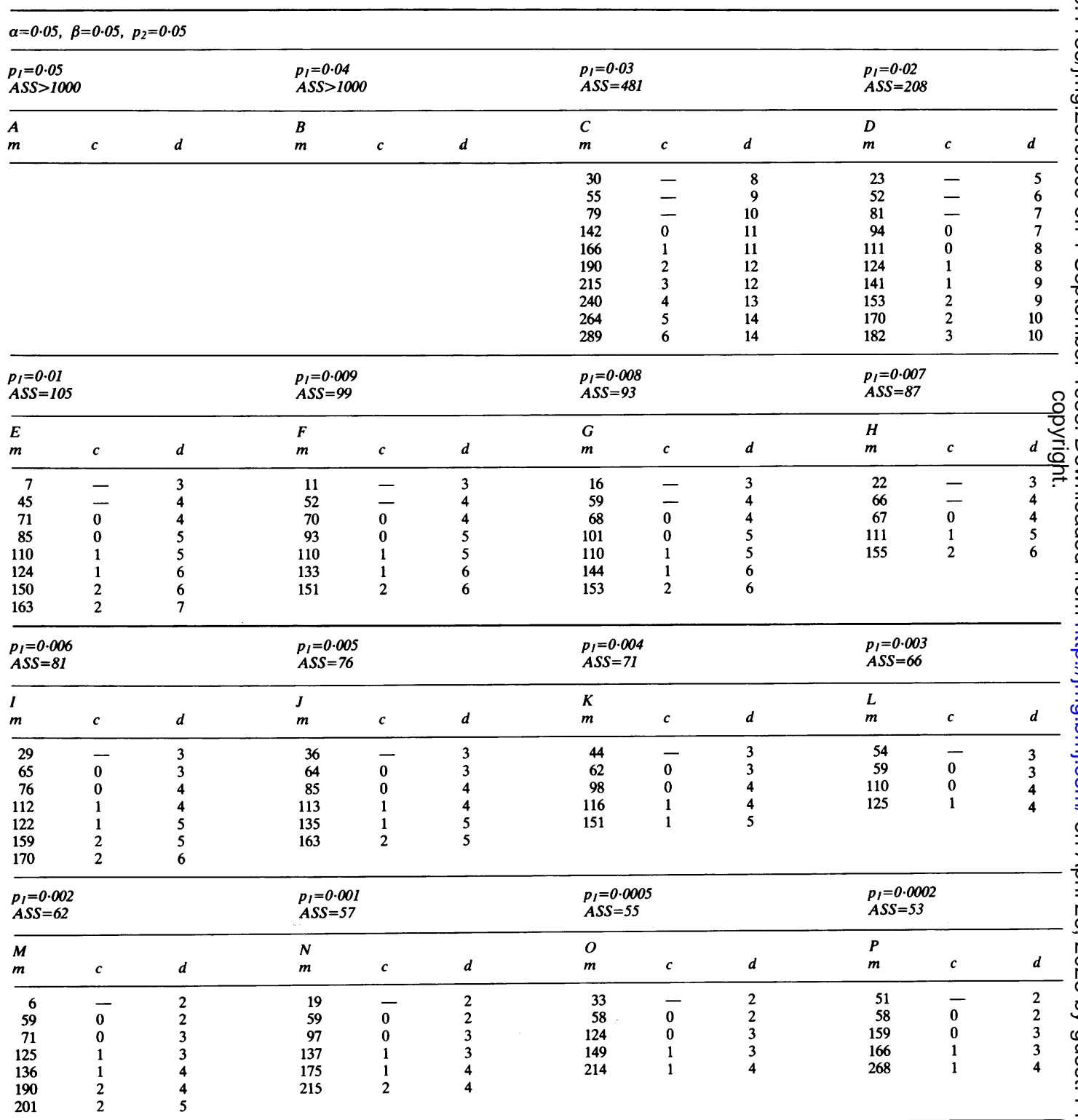

(-) means that no decision can be made as to normal (c) or pathological (d). The tables left blank give average sample sizes that are too large. For interpretation of other symbols, see text. 


\section{Results}

TABLES FOR SEQUENTIAL SAMPLING

Using the previous methodology we have prepared tables 2 to 5 showing the maximum number (c) of abnormal metaphases that can be tolerated in a normal sample (acceptance number), and the minimum number ( $d$, rejection number) that are necessary to diagnose the culture as pathological, when the total number of metaphases sampled by the sequential method is $\mathrm{m}$. The choice of table should be made by reference to table 1 . Tables 2 to 5 are themselves divided in subtables lettered from $\mathrm{A}$ to $\mathrm{P}$ according to different values of $\mathrm{p}_{1}$.

Here we will illustrate the use of the tables in discriminating efficiently between mosaicism and pseudomosaicism in amniotic fluid cultures harvested by the flask method. From the master table 1 we decide for example that we are prepared to let $10 \%$ of cases of mosaicism with less than $10 \%$ abnormal cells go undetected, that is, we settle for table 2. Let us suppose that after 10 cells sampled from the first flask we found two trisomic for chromosome 20, and that we know that in our laboratory the frequency of 'single abnormal (trisomic 20) cells' found with our culture conditions is

TABLE 6 Sequential sampling in the diagnosis of mosaic trisomy 20.

\begin{tabular}{lll}
\hline$\alpha=0.05, \beta=0 \cdot 10$, & $p_{l}=0.00002$, & $p_{2}=0.50$ \\
\hline$m$ & $c$ & $d$ \\
\hline 1 & - & 1 \\
4 & 0 & 1 \\
9 & 0 & 2 \\
16 & 1 & 2 \\
21 & 1 & 3 \\
28 & 2 & 3 \\
\hline
\end{tabular}

ASS $=1$. See text for explanation of the choice of values of $p_{1}$ and $p_{2}$ in this case. $\mathrm{p}_{1}=\mathbf{0 . 0 0 0 2}$, as given by Moller and Ormerod. ${ }^{7}$ Therefore, we will follow table 2P. This table tells us that no diagnosis can be reached unless at least 21 cells have been analysed from a second flask. If after the 21st cell no further trisomy 20 has been found, the case can be reported as normal. If only one +20 cell has been found, the sample cannot be diagnosed as pathological as yet, and further sampling is required. If after a total of 33 cells scored no further +20 metaphases have been found, the sample can be reported as normal. If there were two or more +20 metaphases, the sample can then be reported as mosaic. The procedure would vary depending on the abnormality considered, since $\mathrm{p}_{1}$ could vary for different chromosomes ${ }^{7}$ and also between laboratories. For instance, in the case of suspected mosaicism for an additional fragment $\left(p_{1}=0.003\right.$ in reference 7) we should proceed according to subtable letter L. The tables can be used also to determine the number of colonies from which one metaphase should be analysed if the amniotic fluid was harvested by an in situ method. As a further example let us remember that, according to the experience of Djalali et al, ${ }^{15}$ mosaic trisomy 20 fetuses are at high risk of severe abnormalities only when we find $\mathrm{p}_{2} \geq 50 \%$ fetal cells with that karyotype. We take again $p_{1}=0.0002$ as the frequency with which this karyotype occurs by accident in our culture conditions $^{7}$ and we decide on $\alpha=0.05$ and $\beta=0 \cdot 10$. Substituting these values in the formulae above for $c$ and $d$, and giving successive integer values to $\mathrm{m}$, we obtain table 6 . This table tells us that the earliest decision could be made if having scored one metaphase we found it to be +20 ; the case could then be reported at least as a mosaic of the type defined above. If having scored four metaphases (or colonies) we found no +20 , we could accept the case as normal (or mosaic with $\mathrm{p}_{2} \leq 50 \%$, which according to the experience quoted ${ }^{15}$ would be clinically normal). If we found one or more +20

TABLE 7 Acceptance and rejection numbers in sequential sampling from mixed cell populations. (Example: diagnosis of fragile $X$ in males.)

\begin{tabular}{|c|c|c|c|c|c|c|c|c|c|c|c|c|c|c|}
\hline \multicolumn{3}{|c|}{$\begin{array}{l}p_{2}=0.04 \\
A S S=64\end{array}$} & \multicolumn{3}{|c|}{$\begin{array}{l}p_{2}=0.03 \\
A S S=114\end{array}$} & \multicolumn{3}{|c|}{$\begin{array}{l}p_{2}=0.02 \\
A S S=294\end{array}$} & \multicolumn{3}{|c|}{$\begin{array}{l}p_{2}=0.01 \\
A S S=4160\end{array}$} & \multicolumn{3}{|c|}{$\begin{array}{l}p_{2}=0.001 \\
A S S=7433\end{array}$} \\
\hline$A$ & & & $B$ & & & $C$ & & & $D$ & & & $E$ & & \\
\hline$m$ & $c$ & $d$ & $m$ & $c$ & $d$ & $m$ & $c$ & $d$ & $m$ & $c$ & $d$ & $m$ & $c$ & $d$ \\
\hline 2 & - & 2 & 3 & - & 3 & 3 & - & 3 & 9 & - & 9 & 6 & 6 & - \\
\hline 20 & - & 3 & 66 & - & 4 & 23 & - & 4 & 115 & - & 10 & 60 & 7 & - \\
\hline 67 & 0 & 3 & 97 & 0 & 4 & 104 & - & 5 & & & & & & \\
\hline 73 & 0 & 4 & 130 & 0 & 5 & 171 & 0 & 5 & & & & & & \\
\hline 120 & 1 & 4 & 160 & 1 & 5 & 184 & 0 & 6 & & & & & & \\
\hline 125 & 1 & 5 & 193 & 1 & 6 & & & & & & & & & \\
\hline 172 & 2 & 5 & & & & & & & & & & & & \\
\hline 178 & 2 & 6 & & & & & & & & & & & & \\
\hline
\end{tabular}


colonies, we could report a mosaic. If after nine cells we found only one +20 , we should keep sampling as shown in table 6 . Notice that with these criteria, finding two trisomic cells in 21 does not justify a diagnosis of mosaicism and requires further sampling. Table 6 also tells us that in this particular case the average sample size (ASS) required to reach a diagnosis is one metaphase.

For the fragile $\mathrm{X}$ we have prepared tables 7 and 8 for males and females respectively. Notice that in this case we have settled for the minimum values of $\mathrm{p}_{1}$ given in published reports, which are those of Steinbach et al. ${ }^{2}$ Laboratories should consider whether this value of $p_{1}$ is appropriate in their case. We have prepared subtables according to different values of $p_{2}$, the frequency of expression above which a diagnosis of fragile $X$ positive should be given, which again could vary among laboratories.

Fig 1 shows an example of the effect of abnormal findings in the classification of samples as normal or pathological when the sequential method of sampling is in operation. The process describes a random walk between two parallel lines that define acceptance and rejection zones. When the random walk intersects any of the lines, sampling can cease since a diagnosis can be made.

\section{Discussion}

Having observed the similarities between industrial quality control and cytogenetics when it comes to sampling problems, we have drawn from the methods of the former science to suggest sampling plans for some situations in the latter. Single sampling plans are the simplest to understand, and have the apparent advantage of uniformity since the size is fixed, but they are relatively inefficient in that in most cases they require the largest sample size compared to other procedures. Sequential sampling

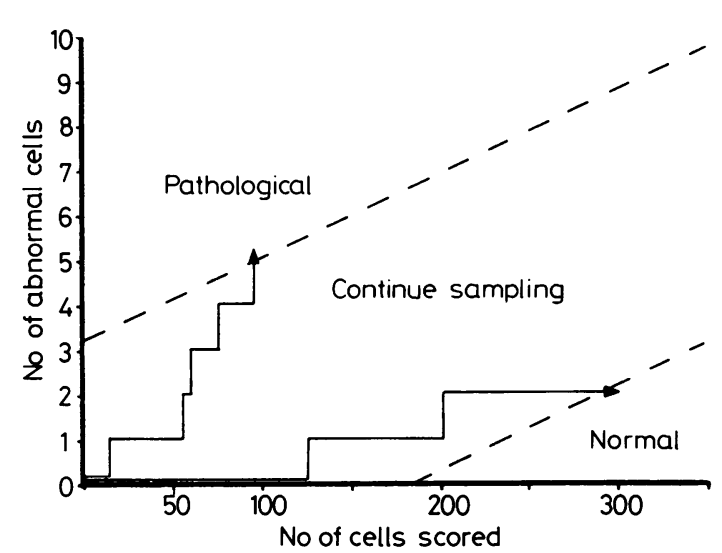

FIG 1 The process of sequential sampling in cytogenetics seen as a random walk between diagnostic lines. A diagnosis is reached as soon as any of the lines is crossed. The figure shows that some 'abnormalities' are compatible with a diagnosis of normal.

appears to be both very suitable for cytogenetic $\stackrel{\mathbb{C}}{-}$ diagnosis and in most cases is also the most efficient $\vec{\bullet}$ procedure.

RELATIVE EFFICIENCIES OF SINGLE, DOUBLEO AND SEQUENTIAL SAMPLING PLANS

For the purpose of comparing the relative efficiencies of equivalent single, double, and sequential plans, we may study the plans for the diagnosis of fragile $X$, using as an example the same constraints for the three procedures, that is, for males $\mathrm{p}_{1}=0.007$ and $1-\alpha=0.95$, and $p_{2}=0.04$ and $\beta=0.10$; and for females $p_{1}=0.015$ and $1-\alpha=0.95$, and $p_{2}=0.04$ and $\beta=0 \cdot 10$. The values of $p_{1}$ are taken from Steinbach et $a l,{ }^{2}$ which are the lowest published values, and $p_{2}$ is chosen at 0.04 as an example. This decision means

TABLE 8 Acceptance and rejection numbers in sequential sampling from mixed cell populations. (Example: diagnosis of fragile $X$ in females.)

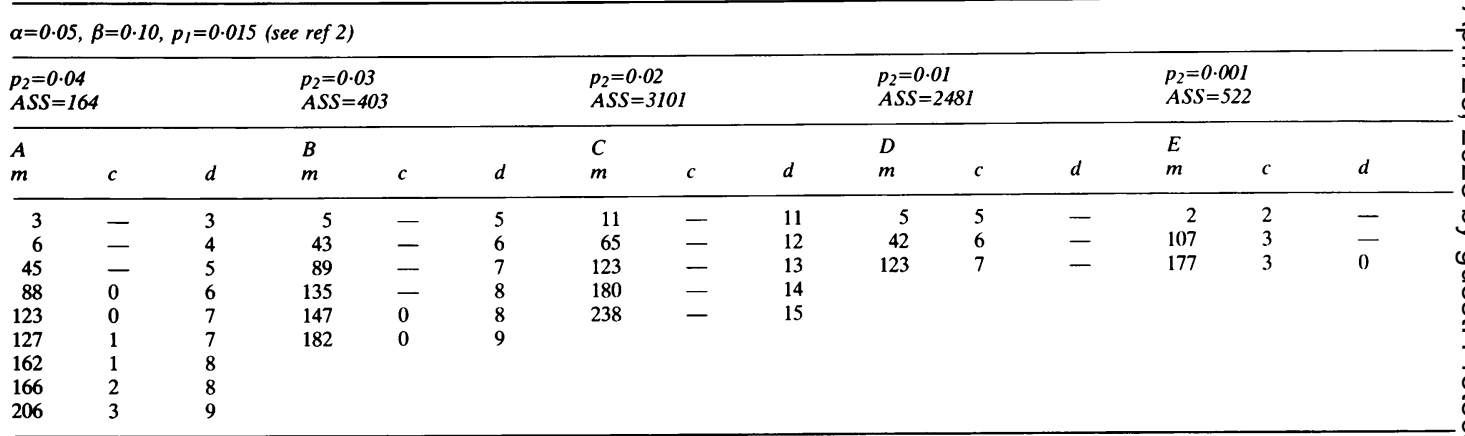


that with these plans the probability of diagnosing as pathological a sample expressing $4 \%$ fragile $X$ will be $90 \%$.

Using these constraints and the tables from Cameron, ${ }^{12}$ we find that the required single sampling plan for males is $117(2,3)$ where 117 is the sample size, 2 is the acceptance number, and 3 the rejection number. This means that if after having scored 117 cells we found two or fewer metaphases with the fragile $X$, the case can be reported as normal. If we found three or more, the case could be reported as pathological. The equivalent plan for females is $313(8,9)$. Using the tables from reference 13 the corresponding double sampling plan for males requires two samples of size 96 , the first one with $\mathrm{c}=0$ and $\mathrm{d}=3$, and if the number of positive cells is $>0$ but $<3$, a second sample of the same size should be drawn with $\mathrm{c}=2$ and $\mathrm{d}=3$. For females, two samples of size 321 , with $c=4$ and $d=10$ for the first and $c=9$ and $d=10$ for the second, are required. Making use of equation (4) above, we find that when we use sequential sampling with those same constraints, we only need to score 64 metaphases as

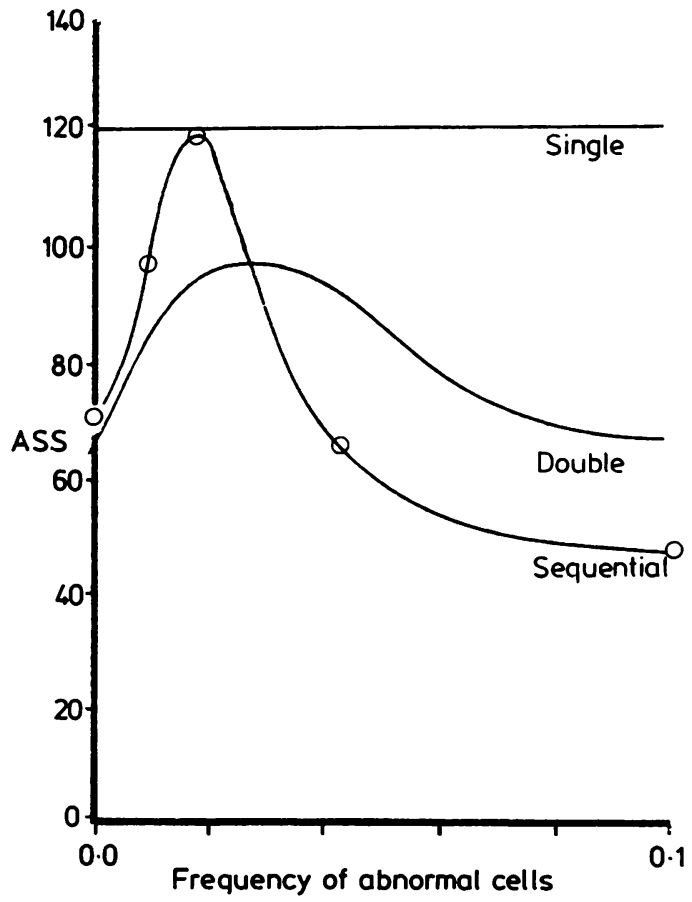

FIG 2 The relative efficiencies of single, double, and sequential sampling in detecting different frequencies of abnormalities (fragile $X$, males). Average sample size (ASS) is lowest in most situations using sequential sampling. an average for males and 187 for females. The five pairs of points (1) to (5) are plotted for these conditions in fig 2 for males and in fig 3 for females. These figures also show the average sample size (ASS) required with single and double sampling plans for the purpose of comparison.

COMPARISON BETWEEN THE TABLES FOR THE EXCLUSION OF MOSAICISM ${ }^{9}$ AND SEQUENTIAL SAMPLING

The problem of sample size in the diagnosis of mixed cell populations has been approached before. ${ }^{89}$ The main difference between sequential sampling as proposed here and the reasoning behind the tables for the exclusion of mosaicism given by Hook $^{9}$ is that we take into account more constraints. Hook's tables are the solutions for $p$ of the first term of the binomial expansion

$$
p^{n}+(1-p)^{n}=\alpha
$$

where $\mathrm{p}$ is the proportion of second cell lines, $\mathrm{n}$ is the number of metaphases of identical karyotype observed, and $\alpha$ is the probability of missing the

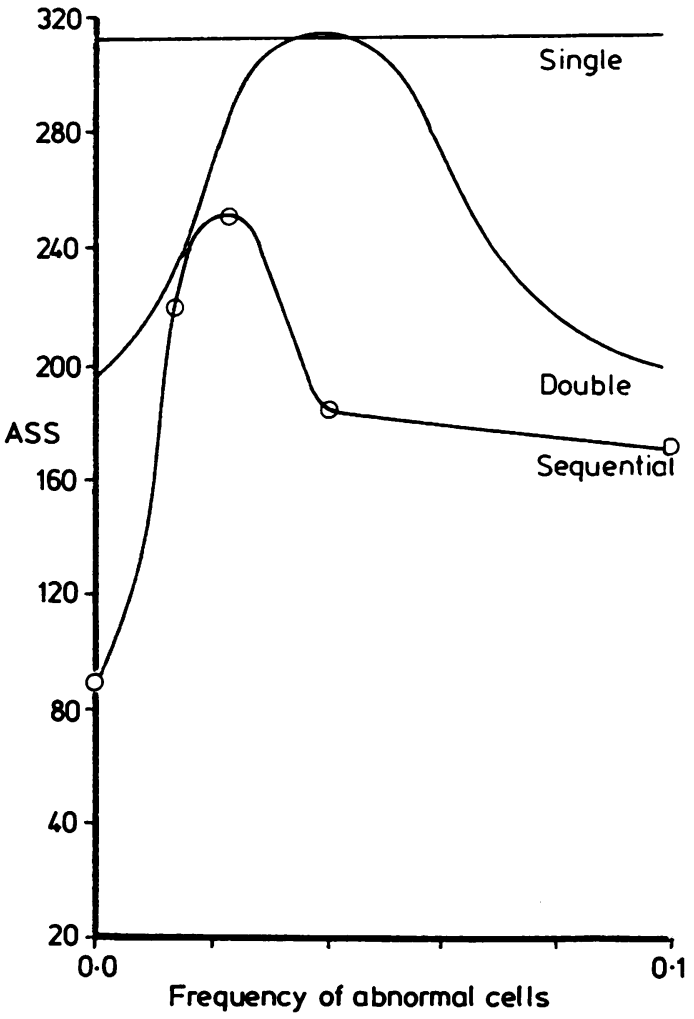

FIG 3 Same as in fig 2, in the case of females with the fragile 
minority cell line present in the proportion of $\mathrm{p} \%$ when $n$ cells have been scored and found identical. With this approach, it is not possible to assess the likelihood that the abnormal findings are attributable to technical factors. Here, we have used evidence available to the effect that in some cases like fragile $X$ or trisomy 20 , the abnormal cells could have two origins, that is, 'genuine pathology' $\left(p_{2}\right)$ or 'technical factors' mimicking those pathological situations $\left(p_{1}\right)$, and we have considered that it would be possible to discriminate between them if they occurred with different frequency; normally we would expect that $p_{1}<p_{2}$. In the case of trisomy 20 in amniotic fluids, this is a reasonable expectation since it is based on the widely different figures given for the frequency of 'single abnormal $(+20)$ cells' $\left(p_{1}=0.0002^{7}\right)$ and the minimum percentage of trisomic cells in the amniotic fluid required for a clinically abnormal outcome $\left(p_{2} \geq 0.5\right) .{ }^{15}$ In the case of other aneuploidies, or the fragile $\mathrm{X}$, there are some data for $\mathrm{p}_{1}$, quoted above in the introduction, but these may vary among laboratories and the minimum frequency required for pathological expression is less well defined. For this reason we have given tables 7 and 8 ordered according to different values of $p_{2}$.

As a final example we will compare the use of Hook's tables and the tables presented here. The problem would be to determine sample size to rule out expression of fragile $\mathrm{X}$ in a male in more than $5 \%$ metaphases with $95 \%$ confidence $(\alpha=0 \cdot 05)$. The use of Hook's table ${ }^{9}$ suggests that this could be done if we do not find any fragile $\mathrm{X}$ in 59 metaphases. A positive finding before that would justify making the hypothesis that the patient expresses in more than $5 \%$ cells, and the sample size would be adjusted accordingly. No further findings would suggest that the patient expresses in a lower proportion, and the sample size would be increased accordingly. For the use of our tables we will consider as before that $\mathrm{p}_{1}=0.007,{ }^{2}$ and we should choose table $3 \mathrm{H}$ which gives the following guidance: no diagnosis can be given before scoring 26 metaphases. If at this stage we have found three or more fragile $\mathrm{X}$, the diagnosis is positive without further sampling. Similarly, sampling can be discontinued if at any stage between 26 and 71 cells we score three or more positives. The case could be reported as negative if at any stage between 51 and 96 cells scored no positive findings have been made. Only one sighting between 96 and 116 metaphases, or only two between 142 and 162 , are not diagnostic of a positive case, but rather could be considered as technical artefacts in an otherwise negative sample. The difference in the advice obtained comes from the fact that, having introduced the concept of acceptance and rejection numbers (or $\mathrm{p}_{1}$ and $\mathrm{p}_{2}$ ), more detailed interpretation $\stackrel{\overparen{D}}{\rightarrow}$ of the findings can be given.

For the proper use of these tables, it is essential $\stackrel{5}{?}$ that individual laboratories, or manufacturers of culture media, investigate the values of $p_{1}$ for the different chromosomes and conditions, and that clinical cytogeneticists find out the minimum pro- $\stackrel{\mathbb{D}}{\Omega}$ portion of abnormal cells that produce noticeable clinical effects $\left(p_{2}\right)$, as discussed above in the case of trisomy 20 and the fragile $\mathrm{X}$.

This work was partly supported by the Central Remedial Clinic, Vernon Avenue, Dublin 3, Ireland. The authors are grateful to Dr David Williams from i the Department of Statistics, University College is Dublin, for helpful comments and for having pointed out some of the references quoted.

\section{References}

1 De Arce MA. Tables for the cytogenetic study of fragile $X \stackrel{\mathbb{D}}{D}$ chromosomes for diagnostic purposes. Clin Genet 1983;24: 320-3.

2 Steinbach P, Barbi G, Boller T. On the frequency of telomeric $\stackrel{乛}{\supset}$ chromosomal changes induced by culture conditions suitable for fragile X expression. Hum Genet 1982;61:160-2.

3 Abuelo D, Castree K, Pueschel S, Padre-Mendoza T, Zolnie\& $\mathrm{K}$. Frequency of fragile $\mathrm{X}$ chromosomes in normal females. Ctim Genet 1985;28:97-100.

4 Vekemans M, Popovich B, Rosenblatt D, Monroe P. Chrom $\bar{q}$ somal breakage in normal and fragile $\mathrm{X}$ subjects using low folare culture conditions. J Med Genet 1983;20:404-7.

5 Nichols P, Martin JM, Kahn J. A mathematical model predicting

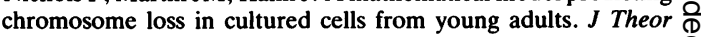
Biol 1978;73:237-45.

6 Galloway SM, Buckton KE. Aneuploidy and ageing: chromo- $\overrightarrow{\bar{O}}$ some studies on a random sample of the population using G- 윽 banding. Cytogenet Cell Genet 1978;20:78-95.

7 Moller P, Ormerod E. Technical and biological aspects of pseudomosaicism and mosaicism. Clin Genet 1987;31:153-7.

8 Ford CE. Mosaics and chimaeras. Br Med Bull 1969:104-9.

9 Hook EB. Exclusion of chromosomal mosaicism: tables of $90 \%$, $95 \%$ and $99 \%$ confidence limits and comments on use. $\mathrm{Am} \mathrm{J}$ Hum Genet 1977;29:94-7.

10 Claussen U, Schafer H, Trampisch HJ. Exclusion of chromosomal mosaicism in prenatal diagnosis. Hum Genet 1984;67: ᄋ 23-8.

11 Wald A. Sequential tests of statistical hypotheses. Ann Math 응 Statist 1945;16:117-23.

12 Cameron JM. Tables for constructing and computing the $D$ operating characteristics of single sampling plans. Ind Qual 음 Control 1952:38.

13 Belz MH. Statistical methods in the process industries. London: MacMillan, 1973.

14 British Standards BS 6001. Sampling procedures. London: $\mathcal{O}$ British Standard Institution, 1972.

15 Djalali M, Steinbach P, Schwinger E, Schwanitz G, Tettenborn $\omega$ $\mathrm{U}$, Wolff $\mathrm{M}$. On the significance of true trisomy 20 mosaicism in amniotic fluid culture. Hum Genet 1985;69:321-6.

Correspondence and requests for reprints to $\mathrm{Dr}$ M A De Arce, Department of Genetics, Faculty of Medicine, University of Navarre, Pamplona, Spain. 